\title{
Inhibition of the MAP kinase activity suppresses estrogen- induced breast tumor growth both in vitro and in vivo
}

\author{
KALADHAR B. REDDY and SELINA GLAROS
}

\author{
Department of Pathology, Wayne State University School of Medicine, \\ The Barbara Ann Karmanos Cancer Institute, Detroit, MI 48201, USA
}

Received July 20, 2006; Accepted October 17, 2006

\begin{abstract}
Elevated expression of mitogen-activated protein kinase (Erk/MAPK) has been noted in a significant percentage of primary human breast cancers. To directly assess the importance of Erk/MAPK activation in estrogen $\left(\mathrm{E}_{2}\right)$-induced tumor progression, we blocked $\mathrm{E}_{2}$-signaling with MEKinhibitor CI-1040 and/or tamoxifen (TAM). Our data show that both MEK-inhibitor CI-1040 and TAM blocked $\mathrm{E}_{2}$ induced MAPK phosphorylation and cell proliferation in MCF-7 breast cancer cells in vitro. However, in vivo studies show that anti-tumor efficacy of combining the CI-1040 and TAM was similar to single agent(s). Furthermore, sequential treatment with TAM followed by CI-1040 or CI-1040 followed by TAM did not significantly reduce $\mathrm{E}_{2}$-induced tumor growth. This suggests that the combination of CI-1040 and TAM may not be synergistic in inhibiting $\mathrm{E}_{2}$-induced tumor growth. However, these findings also indicate that MAPK plays a critical role in $\mathrm{E}_{2}$-induced tumor growth, and that this could be a potential therapeutic target to combat hormonally regulated growth in ER-positive tumors.
\end{abstract}

\section{Introduction}

Multiple lines of evidence have established that the steroid hormone estrogen $\left(E_{2}\right)$ is essential not only for normal breast development but also plays a major role in the development and growth of breast cancer. Approximately three-quarters of all invasive breast tumors are estrogen and/or progesterone receptor-positive $(1,2)$. Endocrine therapy remains the most important systemic treatment in this population of women with hormone receptor-positive breast cancer. It was shown that in estrogen receptor-positive disease, about five years of adjuvant tamoxifen reduces the annual breast cancer death rate by $31 \%$ (3). This benefit is achieved irrespective of the use of chemotherapy, age, progesterone receptor status, or

Correspondence to: Dr Kaladhar B. Reddy, Department of Pathology, Wayne State University School of Medicine, 540 E. Canfield, Detroit, MI 48201, USA

E-mail: kreddy@med.wayne.edu

Key words: estrogen, tamoxifen, CI-1040, MAPK, breast cancer other tumor characteristics. Most endocrine agents act by either blocking the production of estrogen (ovarian ablation and aromatase inhibitors) or the by competing for ER binding (tamoxifen and ICI). The choice of endocrine agent depends on the menopausal status of the patient, because this factor determines the source of estrogen: ovarian or adrenal.

There is an increasing body of evidence, however, that indicates such a therapeutic approach may be somewhat simplistic. Control of breast cancer growth is now perceived to be multifaceted, comprising of an elaborate network of interacting steroid hormone- and growth factor-driven pathways that reinforce individual pathway effects on gene expression $(4,5)$. It has thus been postulated that aberrations in growth factor signal transduction would significantly influence estrogen action $(4,6,7)$. Indeed, it is envisaged that such events may entirely circumvent the cellular requirement for this steroid hormone, supporting tumor growth that is resistant to antihormonal therapies despite retention of functional ER. Of particular interest is the mitogen-activated protein kinase (MAPK), also known as extracellular signal-regulated kinases (Erk1 and Erk2). Experimental studies have demonstrated that MAPKs' are pivotal components of intracellular phosphorylation cascades both in cytoplasm and the nucleus $(8,9)$. Interestingly, activated Erk/MAPK can drive ligand independent activation of ER $(6,10)$. ER-regulated gene expression is subsequently initiated, as has been demonstrated using estrogen response element-containing reporter gene constructs transiently transfected in vitro $(4,11)$. In addition, elevated Erk/MAPK has been demonstrated to occur within some breast tumors (12-14). Finally, changes in several key regulatory pathways such as ErbB, insulin-like growth factor receptor, and protein kinase $\mathrm{C}$ leading to Erk/MAPK activation are frequently elevated in clinical breast tumors, and these are associated with anti-hormonal resistance (15-18). In this study we investigated whether mitogen-activated extracellular signal regulated kinase kinase (MEK) inhibitor, CI-1040 used as a monotherapy or in combination with anti-estrogen TAM, is more efficient in inhibiting breast tumor growth both in vitro and in vivo.

\section{Materials and methods}

Materials. Human breast cancer MCF-7 cells were purchased from American Type Culture Collection. The cells were grown in DMEM supplemented with $10 \mathrm{ng} / \mathrm{ml}$ insulin, 5\% fetal calf serum, and antibiotics (penicillin/streptomycin). DMEM, 
phenol red-free DMEM, recombinant human insulin, and fetal bovine serum were purchased from Invitrogen (Carlsbad, CA). Charcoal-treated fetal bovine serum was purchased from Cocalico Biologicals, PA. The 17ß-estradiol $\left(E_{2}\right)$ and 4-hydroxytamoxifen, RNase, Cremophore EL were purchased from Sigma, CI-1040 was provided by Pfizer Global Research and Development (Groton, CT). The 17ß-estradiol pellets (0.72-mg/biodegradable carrier-binding pellet) and TAM pellets $(5 \mathrm{mg} /$ biodegradable carrier-binding pellet) were purchased from Innovative Research of America (Sarasota, FL). Phospho-MAPK antibody was purchased from New England Biolabs, MA. Anti-MAPK antibody was purchased from Zymed Laboratories, CA. ER antibody was purchased from Dako (Carpinteria, CA) and NeoMarkers.

Western immunoblot analysis. Western blotting was performed as described previously using a standard protocol (19). Crude protein extracts were obtained by lysing $5 \times 10^{6}$ cells in a buffer [50 mM Tris- $\mathrm{HCl}$ (pH 7.6), 1\% NP-40, 2 mM EDTA, $0.5 \% \mathrm{Na}$ deoxycholate, $150 \mathrm{mM} \mathrm{NaCl}, 1 \mathrm{mM} \mathrm{Na}$ orthovanadate, $2 \mathrm{mM}$ EGTA, $4 \mathrm{mM}$ Na p-Nitro phenyl phosphate, $100 \mathrm{mM}$ Na fluoride] supplemented with protease inhibitors [leupeptin $(0.5 \%)$, aprotinin $(0.5 \%)$, and PMSF (0.02\%)]. Samples containing $50 \mu \mathrm{g}$ of total protein were electrophoresed on $7.5 \%$ (for EGFR) and 10\% (for MAPK) SDS-polyacrylamide gels and transferred on to nitrocellulose membrane by electroblotting. Membranes were probed with antibodies, followed by HRP-conjugated mouse or rabbit secondary antibodies (Amersham) and enhanced chemiluminescence detection (Amersham). For quantification of activity, band intensities of the phospho- and total protein(s) were quantified using Biorad 'Quantity one' software.

Monolayer growth assay. Cells were plated in 6-well tissue culture plates, at a density of $1 \times 10^{5}$ cells/well, in DMEM containing 5\% FCS. After $24 \mathrm{~h}$, the seeding medium was removed, cells were washed twice with PBS, and the medium was replaced with phenol red-free and serum-free DMEM with $\mathrm{E}_{2}$, Tam and with or without CI-1040. The medium was replaced with fresh medium, containing the same supplements, on day 3. The cells were counted in triplicate after being suspended in PBS with $1 \mathrm{mM}$ EDTA, on day 5.

Animal efficacy studies. MCF-7 cells ( $1 \times 10^{6}$ cells/mouse) were suspended in matrigel and injected subcutaneous (s.c.) in ovariectomized female SCID-beige mice 5-6 weeks old (Taconic Farms, Germantown, NY). The estrogen pellets (17ßestradiol - $0.72 \mathrm{mg} /$ biodegradable carrier-binder pellet) was implanted s.c. at the time of cell inoculation. Treatment (CI1040) was administered by gavage and/or tamoxifen pellet ( 5 $\mathrm{mg} /$ biodegradable carrier-binder pellet) was implanted s.c. one day after tumor cell implantation. The $\mathrm{E}_{2}$ and TAM slow release pellets were obtained from Innovative Research of America. The CI-1040 (PD 184352) was prepared in a vehicle of $10 \%$ Cremophore EL (Sigma), $10 \%$ ethanol and $80 \%$ water. Ten animals were included in each group. Tumor size was evaluated periodically by caliper measurements, generally twice per week. Tumor size measurement of the length (a) and width (b) of tumor and volumes calculated as $\left(\mathrm{a} \mathrm{b} \mathrm{b}^{2}\right) / 2$.

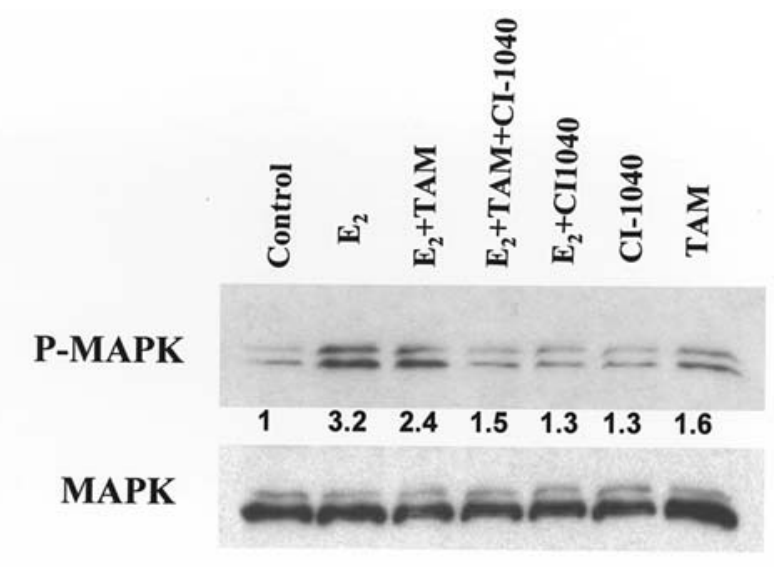

Figure 1. Effect of CI-1040 on estrogen-induced Erk/MAPK phosphorylation in MCF-7 cells. Cells were grown as shown in Fig. 2 in the presence of estrogen and/or tamoxifen (TAM), in the presence or absence of $10 \mu \mathrm{M} \mathrm{CI}-$ 1040. Erk/MAPK was evaluated by Western blot analysis.

\section{Results}

MEK-inhibitor CI-1040 blocks $E_{2}$-induced MAPK phosphorylation and cell proliferation in vitro. CI-1040 is an oral inhibitor of mitogen-activated extracellular signal regulated kinase kinase (MEK), a key enzyme in the Ras-Raf-MEKMAPK (Erk) pathway known to be involved in key cellular activities including proliferation, differentiation, and apoptosis (20-22). To confirm that these compounds can block MAP kinase phosphorylation in breast cancer cells, we examined the effect of CI-1040 on MCF-7 breast cancer cells. After treating the cells with estrogen $\left(\mathrm{E}_{2}\right)$ and/or TAM in the presence or absence of CI-1040, Western blot analysis using phospho-MAPK antibodies was performed. The data show that $\mathrm{E}_{2}\left(10^{-8} \mathrm{M}\right)$ significantly increases phosphorylated MAPK levels compared to control cells. $E_{2}$-induced phosphorylated MAPK levels were inhibited by TAM and CI-1040. However, maximum inhibition of phosphorylated Erk/MAPK was seen in the presence of MEK-inhibitor CI-1040 (Fig. 1).

To investigate the effect of CI-1040 on $\mathrm{E}_{2}$-stimulated cell proliferation, we examined its effects on MCF-7 cell. In MCF-7 cells $\mathrm{E}_{2}$-induced 2 -fold increase in cell proliferation, and $\mathrm{E}_{2}$-induced cell proliferation was significantly inhibited by TAM $\left(10^{-6} \mathrm{M}\right)$ and CI-1040 (10 $\left.\mu \mathrm{M}\right)$ (Fig. 2). At concentrations of $20 \mu \mathrm{M}, \mathrm{CI}-1040$ inhibited cell growth significantly below controls (data not shown), suggesting that, at this concentration, CI-1040 might inhibit other growth-regulatory processes, in addition to its effect on MEK mediated growth. Cells growing under these conditions in the presence of CI-1040 $(10 \mu \mathrm{M})$ appeared to remain viable, as indicated by their morphological appearance, by the lack of floating cells, and by the ability of the cells to exclude trypan blue ( $>90 \%$ viable). These data suggested that the inhibitory effect of the CI-1040 was cytostatic rather than cytotoxic at this concentration.

Inhibition of MAPK signaling by CI-1040 inhibits estrogeninduced tumor growth in vivo. Exposure to CI-1040 and/or TAM inhibited on $\mathrm{E}_{2}$-induced cell proliferation in vitro, we tested the ability of CI-1040 to inhibit $\mathrm{E}_{2}$-mediated tumor 


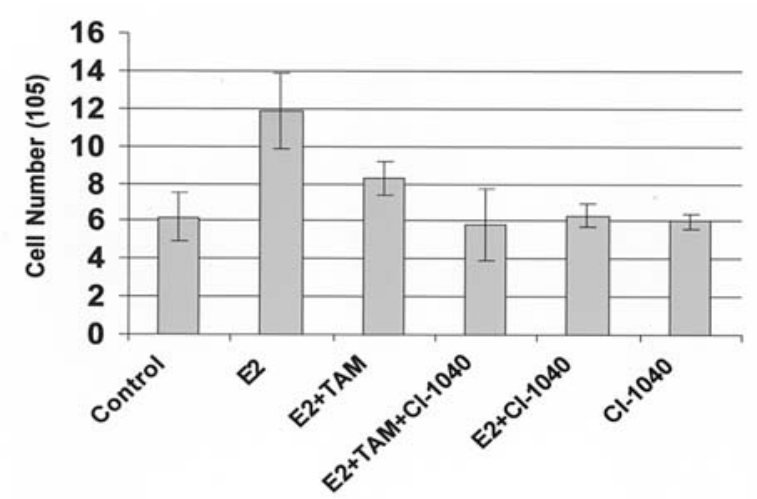

Figure 2. Effect of MEK-inhibitor CI-1040 on estrogen-stimulated cell proliferation. MCF-7 cells were grown in DMEM with serum to $50-60 \%$ confluence. The seeding medium was removed, cells were washed twice with PBS and the medium was replaced with phenol red-free DMEM with 5\% charcoal stripped serum. The medium was replaced with fresh medium on day 3 . The cells were counted on day 5 . Bars, mean \pm SE of triplicate determinations.

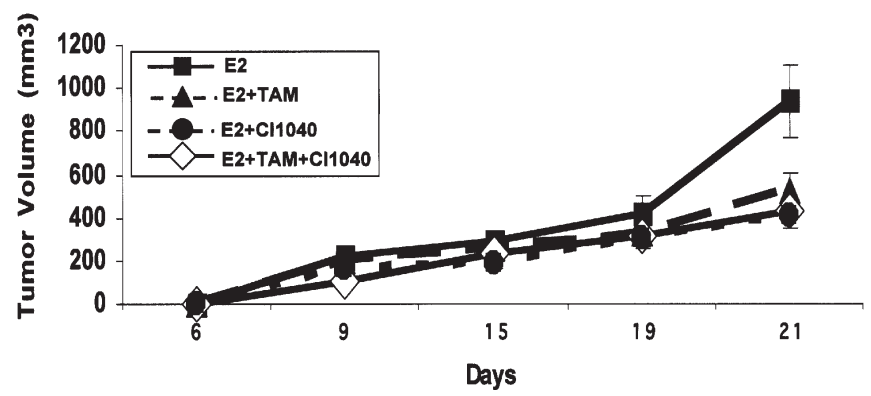

Figure 3. Anti-tumor activity of CI-1040 in estrogen-mediated breast tumors. MCF-7 cells were injected subcutaneously into the mice and randomly divided into 4 groups. All the mice were given estrogen. First group of mice were given tamoxifen (TAM), the second group was given CI-1040 by oral gavage, third group was given TAM and CI-1040 and the fourth group were given estrogen and these served as the control group. When compared to estrogen treated mice, both TaM and CI-1040 significantly inhibited tumor growth by 21 days $(\mathrm{p}<0.005)$. However, combination of TAM plus CI-1040 was unable to further reduced the $\mathrm{E}_{2}$-induced tumor growth when compared to TAM alone or CI-1040 alone treated group. Data presented are mean \pm SE.

growth in vivo, either with or without TAM. MCF-7 cells were injected (s.c.) into 5-6 weeks old female ovariectomized SCID mice, which were implanted with $\mathrm{E}_{2}$-pellet (17ß-estradiol - $0.72 \mathrm{mg} /$ biodegradable carrier-binder pellet, 90-day slow release pellet). Ten animals per treatment group were used. One day after the injection of the cells the mice were given CI$1040(154 \mathrm{mg} / \mathrm{kg})$ by oral gavage and/or TAM for 21 days. The dosage of CI-1040, estrogen and TAM was determined from previous studies $(4,22)$. Our data show that mice receiving estrogen plus CI-1040 or estrogen plus TAM had significantly smaller tumors compared to estrogen-treated mice $(\mathrm{p}<0.05)$ (Fig. 3). However, concurrent administration of $\mathrm{E}_{2}, \mathrm{CI}-1040$, and TAM did not show additive effect on inhibition of tumor growth. Phospho-MAPK levels in TAM and/or CI-1040 were significantly lower compared to $\mathrm{E}_{2}$-treated tumors (Fig. 4). However, differences with in the tumors either treated with TAM or CI-1040 or a combination of TAM and CI-1040 are not significantly different.

To determine if sequential treatment, with TAM followed by CI-1040 or reverse sequence will enhance the inhibitory

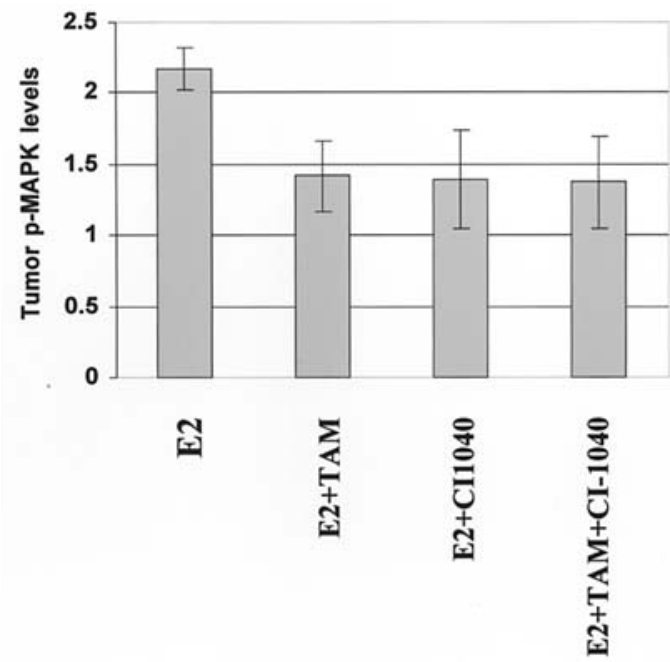

Figure 4. Effect of CI-1040 and tamoxifen (TAM) on estrogen-induced Erk/MAPK phosphorylation in vivo. Protein from MCF-7 tumors was evaluated by Western blot analysis. Data shown represent mean \pm SE of all the tumors in each group.
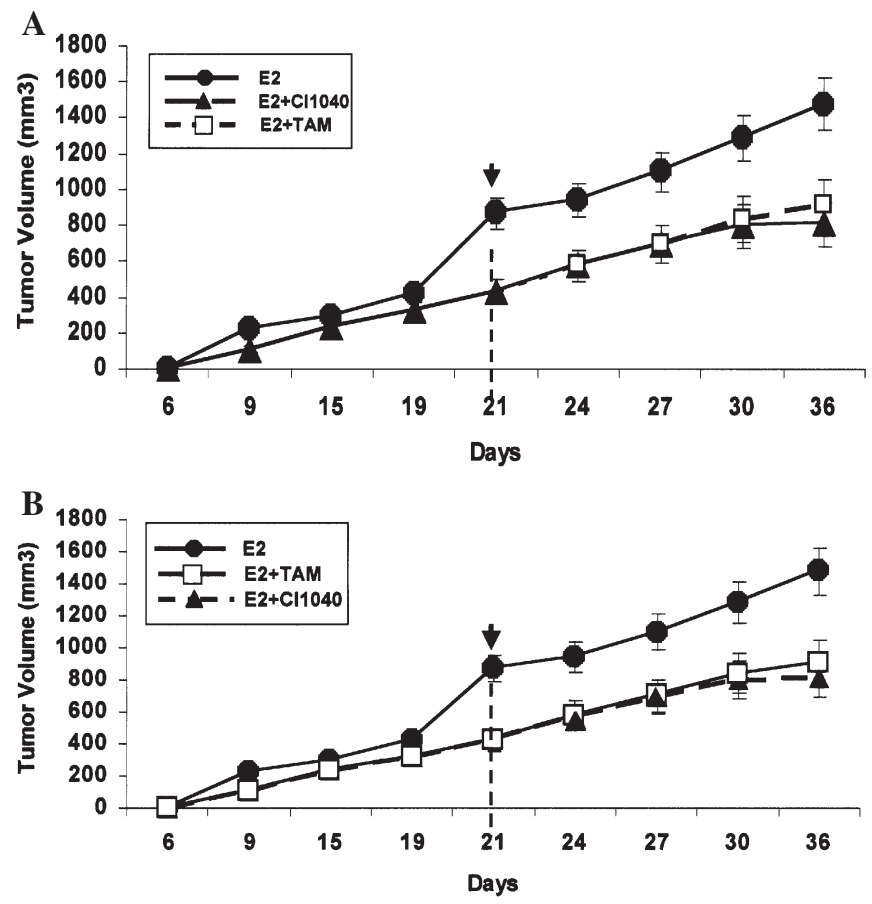

Figure 5. Anti-tumor activity of CI-1040 and tamoxifen (TAM) in estrogenmediated breast tumors when treated sequentially. MCF-7 cells were injected subcutaneously into the mice and randomly divided into two groups. All the mice were given estrogen. One group of ten mice was given CI-1040 for 21 days and then mice were divided into two groups of five mice each. First group was continued on CI-1040, whereas the other group was given TAM for another 15 days (A). (B), One group of ten mice were given TAM for 21 days and then mice were divided into two groups of five mice. One group was continued on TAM, whereas the other group was given CI-1040 for another 15 days. However, sequential treatment with CI1040 followed by TAM or TAM followed by CI-1040 was unable to further reduced the $\mathrm{E}_{2}$-induced tumor growth when compared to TAM alone or CI1040 alone treatment groups. Data presented are mean \pm SE.

effects of the drug compared to concurrent administration of CI-1040 and TAM. We conducted in vivo experiments were ten mice were first treated with CI-1040 for 21 days and then 
they were split into two groups (average tumor size in both the groups is roughly the same): one group of five mice continued to get CI-1040 and the other group of five mice received TAM (Fig. 5A). In the second treatment group ten mice were first treated with TAM for 21 days and then they were split into two groups (average tumor size in both the groups is roughly the same): one group of five mice continued to get TAM and the other group of five mice received CI-1040 (Fig. 5B). Our data do not show any significant advantages in sequential treatment compared to individual treatment with CI-1040 or TAM alone in vivo.

\section{Discussion}

In breast cancer, the steroid hormone estrogen is one of the most potent mitogens. Although it is generally believed that MAPK are activated in response to signals generated by cell surface receptors, it was recently reported that estrogen and progestin activate MAPK signaling in human breast cancer cell lines (24). However, this activation is most likely not mediated by nuclear steroid receptors, but by cell surface forms of estrogen receptors. Furthermore, secretion of a growth factor upon estrogen stimulation of human breast cancer cells can also lead to autocrine/paracrine activation of MAPK $(19,25,26)$. The Erk/MAPK signaling cascade is intimately involved in mediating proliferative signals for receptors whose overexpression and/or constitutive activation has been shown to play an important role in the pathogenesis and progression of breast cancer. Given the complexity of this signaling network and proliferative effect, therapeutic blocking of MEK, as key mediator of $E_{2}$-mediated cell proliferation, could have significant clinical benefit in the treatment of breast cancer. To test this hypothesis we determined the role of Erk/MAPK in $\mathrm{E}_{2}$-induced cell proliferation both in vitro and in vivo models. We found that TAM partially blocked $\mathrm{E}_{2}$-induced MAPK activity and cell proliferation in vitro. However, addition of MEK-inhibitor CI-1040, inhibited most of the estrogen-induced MAPK activity. However, both TAM and CI-1040 inhibited $\mathrm{E}_{2}$ induced cell proliferation in MCF-7 human breast cancer cells. These experiments demonstrated an essential role of MEK/MAPK signaling for the maintenance of $\mathrm{E}_{2}$-induced cell proliferation in vitro.

Our data show that in in vivo tumor models both TAM and CI-1040 as single agents, showed statistically significant reduction in $\mathrm{E}_{2}$-induced tumor volume. The anti-tumor efficacy of combined TAM and MEK-inhibitor CI-1040 was similar to single agents. We further tested to see if sequential treatment, TAM followed by CI-1040 or reverse sequence CI1040 followed by TAM would have additive effect on reducing the tumor volume. However, sequential treatment did not significantly reduced the tumor volume compared to single agents. This suggests that either combination or sequential treatment with TAM and CI-1040 are ineffective in increasing efficacy, relative to either single agent that was associated with suppressed proliferation. One of the reasons may be that TAM competes with $\mathrm{E}_{2}$ to bind to ER $\alpha$ and decrease the synthesis and secretion of growth factors such as $\mathrm{TGF} \alpha, \mathrm{HRG}, \mathrm{IGF}-\mathrm{II}$ and this in turn reduce MAPK activity by autocrine and/or paracrine mechanism $(9,26)$. CI-1040 also blocks MAPK activity; therefore combining TAM and CI-1040 may not be effective because both TAM and CI1040 may be blocking $\mathrm{E}_{2}$-induced Erk/MAPK activity.

A majority of primary human breast cancers are considered estrogen receptor $(E R \alpha)$-positive, and ER expression in breast tumors provides prognostic information, in addition to treatment response information (27). Targeting the ER using the selective ER modulator, tamoxifen, is efficacious in both the treatment and prevention of human breast cancer $(28,29)$. However, despite tamoxifen therapy success, some primary ER $\alpha$-positive tumors do not respond to TAM (30). In these cases inhibition of MEK may be used to prevent $\mathrm{E}_{2}$-induced tumor growth. However, phase II results using CI-1040, in breast, colon, and non-small cell lung cancer demonstrated insufficient CI-1040 anti-tumor activity (31). However, in these studies baseline tumor biopsies were not obtained for testing of phospho-MAPK expression in this study. Instead, archived tumor specimens available from an original diagnostic or a more recent biopsy were assessed. Therefore, it is possible that tumor specimens may underreport the true levels of constitutive expression present at the time of the original biopsy. Recent clinical data highlight the difficulty in identifying the patients who are most likely to respond to treatment with molecularly targeted agents (32). With the central role of MEK in mediating multiple oncogenic signaling pathways, inhibitors of this molecular target have the potential to have a broad spectrum of anti-tumor activity.

Although activation of the ER by Erk/MAPK has been implicated as a mechanism of resistance to anti-estrogen therapies $(33,34)$. Recent data from our group showed that Erk/MAPK induced ER $\alpha$ activation lowers $\mathrm{E}_{2}$-requirement for optimal ER-signaling and accelerates $\mathrm{E}_{2}$-independent tumor growth without diminishing sensitivity to the inhibitory effects of anti-estogens (4). Consistent with our observation Murphy et al have shown that Erk/MAPK-induced ER phosphorylation is associated with better disease outcome in women treated with TAM $(35,36)$. These data suggest that Erk/MAPK phosphorylation plays a major role in breast cancer progression. Other studies have reported that receptor blocking antibodies and an inhibitor of Erk/MAPK can also inhibit growth of HER-2 overexpressing breast tumor cells when given together with TAM $(33,34)$. However, our data show that anti-tumor efficacy of combined TAM and MEK-inhibitor CI-1040 was similar to single agents in vivo. Given the complexity of this signaling network and important proliferative effect of coactivation of multiple growth promoting pathways, the therapeutic blockade of MEK, could have significant clinical benefit in the treatment of $\mathrm{E}_{2}$-induced breast cancer.

\section{Acknowledgements}

This work was supported in part by NIH Grant R01 CA 83964 (to K.B.R.) and Department of Pathology, WSU.

\section{References}

1. Buzdar AU and Hortobagyi G: Update on endocrine therapy for breast cancer. Clin Cancer Res 4: 527-534, 1998.

2. Huang HJ, Neven P, Drijkoningen M, et al: Association between HER-2/neu and the progesterone receptor in oestrogen-dependent breast cancer is age-related. Breast Cancer Res Treat 91: 81-87, 2005. 
3. Early Breast Cancer Trialists' Collaborative Group (EBCTCG): Effects of chemotherapy and hormonal therapy for early breast cancer on recurrence and 15-year survival: an overview of the randomised trials. Lancet 365: 1687-1717, 2005.

4. Atanaskova N, Keshamouni VG, Krueger JS, Schwartz JA, Miller F and Reddy KB: MAP kinase/estrogen receptor cross-talk enhances estrogen-mediated signaling and tumor growth but does not confer tamoxifen resistance. Oncogene 21: 4000-4008, 2002.

5. Nicholson RI and Gee JM: Oestrogen and growth factor crosstalk and endocrine insensitivity and acquired resistance in breast cancer. Br J Cancer 82: 501-513, 2000.

6. Kato S, Endoh H, Masuhiro Y, et al: Activation of the estrogen receptor through phosphorylation by mitogen-activated protein kinase. Science 270: 1491-1494, 1995.

7. Kelly MJ and Levin ER: Rapid actions of plasma membrane estrogen receptors. Trends Endocrinol Metab 12: 152-156, 2001.

8. Canagarajah BJ, Khokhlatchev A, Cobb MH and Goldsmith EJ: Activation mechanism of the MAP kinase ERK2 by dual phosphorylation. Cell 90: 859-869, 1997.

9. Keshamouni VG, Mattingly RR and Reddy KB: Mechanism of 17-beta-estradiol-induced Erk1/2 activation in breast cancer cells. A role for HER2 and PKC-delta. J Biol Chem 277: 22558-22565, 2002.

10. Bunone G, Briand PA, Miksicek RJ and Picard D: Activation of the unliganded estrogen receptor by EGF involves the MAP kinase pathway and direct phosphorylation. EMBO J 15: 2174-2183, 1996.

11. Coutts AS and Murphy LC: Elevated mitogen-activated protein kinase activity in estrogen-nonresponsive human breast cancer cells. Cancer Res 58: 4071-4074, 1998.

12. Maemura M, Iino Y, Koibuchi Y, Yokoe T and Morishita Y: Mitogen-activated protein kinase cascade in breast cancer. Oncology 57 (Suppl 2): 37-44, 1999.

13. Mueller H, Flury N, Eppenberger-Castori S, Kueng W, David F and Eppenberger U: Potential prognostic value of mitogenactivated protein kinase activity for disease-free survival of primary breast cancer patients. Int J Cancer 89: 384-388, 2000.

14. Sivaraman VS, Wang H, Nuovo GJ and Malbon CC: Hyperexpression of mitogen-activated protein kinase in human breast cancer. J Clin Invest 99: 1478-1483, 1997.

15. Houston SJ, Plunkett TA, Barnes DM, Smith P, Rubens RD and Miles DW: Overexpression of c-erbB2 is an independent marker of resistance to endocrine therapy in advanced breast cancer. $\mathrm{Br}$ J Cancer 79: 1220-1226, 1999.

16. Nabha SM, Glaros S, Hong M, et al: Upregulation of PKC-delta contributes to antiestrogen resistance in mammary tumor cells. Oncogene 24: 3166-3176, 2005.

17. Nicholson RI, McClelland RA, Finlay P, et al: Relationship between EGF-R, c-erbB-2 protein expression and Ki67 immunostaining in breast cancer and hormone sensitivity. Eur J Cancer 29A: 1018-1023, 1993.

18. Rocha RL, Hilsenbeck SG, Jackson JG, et al: Insulin-like growth factor binding protein-3 and insulin receptor substrate-1 in breast cancer: correlation with clinical parameters and disease-free survival. Clin Cancer Res 3: 103-109, 1997.

19. Reddy KB, Mangold GL, Tandon AK, et al: Inhibition of breast cancer cell growth in vitro by a tyrosine kinase inhibitor. Cancer Res 52: 3636-3641, 1992.
20. Cowley S, Paterson H, Kemp P and Marshall CJ: Activation of MAP kinase kinase is necessary and sufficient for PC12 differentiation and for transformation of NIH 3T3 cells. Cell 77: 841-852, 1994.

21. Mansour SJ, Matten WT, Hermann AS, et al: Transformation of mammalian cells by constitutively active MAP kinase kinase. Science 265: 966-970, 1994.

22. Sebolt-Leopold JS, Dudley DT, Herrera R, et al: Blockade of the MAP kinase pathway suppresses growth of colon tumors in vivo. Nat Med 5: 810-816, 1999.

23. Pages G, Lenormand P, L'Allemain G, Chambard JC, Meloche S and Pouyssegur J: Mitogen-activated protein kinases p42mapk and p44mapk are required for fibroblast proliferation. Proc Natl Acad Sci USA 90: 8319-8323, 1993.

24. Castoria G, Barone MV, Di Domenico M, et al: Non-transcriptional action of oestradiol and progestin triggers DNA synthesis. EMBO J 18: 2500-2510, 1999.

25. Kaulsay KK, Mertani HC, Tornell J, Morel G, Lee KO and Lobie PE: Autocrine stimulation of human mammary carcinoma cell proliferation by human growth hormone. Exp Cell Res 250: 35-50, 1999.

26. Reddy KB, Yee D, Hilsenbeck SG, Coffey RJ and Osborne CK: Inhibition of estrogen-induced breast cancer cell proliferation by reduction in autocrine transforming growth factor alpha expression. Cell Growth Differ 5: 1275-1282, 1994.

27. Osborne CK: Steroid hormone receptors in breast cancer management. Breast Cancer Res Treat 51: 227-238, 1998.

28. Fisher B, Costantino J, Redmond C, et al: A randomized clinical trial evaluating tamoxifen in the treatment of patients with nodenegative breast cancer who have estrogen-receptor-positive tumors. N Engl J Med 320: 479-484, 1989.

29. Fisher B, Costantino JP, Wickerham DL, et al: Tamoxifen for prevention of breast cancer: report of the National Surgical Adjuvant Breast and Bowel Project P-1 Study. J Natl Cancer Inst 90: 1371-1388, 1998.

30. Clarke R, Leonessa F, Welch JN and Skaar TC: Cellular and molecular pharmacology of antiestrogen action and resistance. Pharmacol Rev 53: 25-71, 2001.

31. Rinehart J, Adjei AA, Lorusso PM, et al: Multicenter phase II study of the oral MEK inhibitor, CI-1040, in patients with advanced non-small-cell lung, breast, colon, and pancreatic cancer. J Clin Oncol 22: 4456-4462, 2004.

32. Burton A: What went wrong with Iressa? Lancet Oncol 3: 708, 2002.

33. Kurokawa H, Lenferink AE, Simpson JF, et al: Inhibition of HER 2/neu (erbB-2) and mitogen-activated protein kinases enhances tamoxifen action against HER2-overexpressing, tamoxifen-resistant breast cancer cells. Cancer Res 60: 5887-5894, 2000 .

34. Witters LM, Kumar R, Chinchilli VM and Lipton A: Enhanced anti-proliferative activity of the combination of tamoxifen plus HER-2-neu antibody. Breast Cancer Res Treat 42: 1-5, 1997.

35. Murphy L, Cherlet T, Adeyinka A, Niu Y, Snell L and Watson P: Phospho-serine-118 estrogen receptor-alpha detection in human breast tumors in vivo. Clin Cancer Res 10: 1354-1359, 2004.

36. Murphy LC, Niu Y, Snell L and Watson P: Phospho-serine-118 estrogen receptor-alpha expression is associated with better disease outcome in women treated with tamoxifen. Clin Cancer Res 10: 5902-5906, 2004 